\title{
ESTUDO COMPARATIVO ENTRE DUAS ANOMALIAS MAGNÉTICAS DE CORPOS ALCALINOS NO ALTO DE CABO FRIO E EM ÁREAS PROXIMAIS DA BACIA DE SANTOS APOIADO POR DECONVOLUÇÃO DE EULER
}

\author{
André Etienne Pacifico Peçanha Demonte Ferraz ${ }^{1}$, Adalberto da Silva² e André Luiz Ferrari ${ }^{3}$ \\ Recebido em 21 setembro, 2007 / Aceito em 26 novembro, 2008 \\ Received on September 21, 2007 / Accepted on November 26, 2008
}

\begin{abstract}
The analysis of aeromagnetic data from the continental shelf area near the Santos Basin border shows several punctual anomalies that can be related to alkaline bodies as observed in the adjacent onshore area. In this paper we propose a comparative analysis of the magnetic anomalies associated to the well know onshore Arraial do Cabo (Cabo Frio, Rio de Janeiro, Brazil) alkaline bodies and a prominent anomaly located near the Santos Basin Cretaceous hinge line. Here we propose a non-conventional workflow applying the 2D Euler deconvolution method integrated in a routine that honors information derived from the analytical signal amplitudes and that embodies two distinct criteria to compute and analyze the solutions. Also a routine to directly estimate the parameters was implemented in order to compare with the solutions obtained by applying those criteria. The resulting solutions can be used as additional information to constrain other inversion methods and to support more realistic geological models in areas lacking direct observations. The estimated physical parameters derived from local alkaline bodies that cause the observed onshore and offshore anomalies can favor a global comparative study concerning their magnetic signatures. Ongoing research dealing with these alkaline intrusions and the accompanying magnetic anomalies will be relevant to understand the magmatic and tectonic events associated with the Santos Basin evolution.
\end{abstract}

Keywords: Euler deconvolution, high resolution airborne magnetic data, Cabo Frio High, Santos Basin, alkaline magmatism.

RESUMO. Diversas anomalias isoladas são observadas a partir de dados aeromagnetométricos na região da plataforma continental adjacente à Bacia de Santos. Estas anomalias são associadas a intrusões alcalinas por comparação com as anomalias de corpos aflorantes conhecidos. Neste trabalho, é apresentado um estudo comparativo entre a resposta magnética dos corpos alcalinos aflorantes ou subaflorantes conhecidos na região de Arraial do Cabo (Cabo Frio, RJ) e uma notável anomalia que ocorre na área submersa, próxima à charneira cretácea da Bacia de Santos. Alicerçando este estudo, é proposto o método da deconvolução de Euler 2D abordado não da forma clássica, mas sim programado para funcionar integrado à amplitude do sinal analítico e utilizar três critérios distintos de cálculo para análise das soluções. Soluções obtidas com esta metodologia podem ser utilizadas como informação adicional para vincular outros métodos de inversão, objetivando a criação de modelos mais realistas que reflitam o ambiente geológico em regiões carentes de informação direta. Os parâmetros físicos estimados para diversos corpos alcalinos locais, causadores das anomalias observadas tanto na região submersa como na área emersa, podem vir a subsidiar um estudo comparativo global sobre seu comportamento magnético. A ampliação do conhecimento sobre as intrusões alcalinas e suas respostas magnéticas, contribuirá para o entendimento de importantes eventos magmáticos e tectônicos ligados à evolução da Bacia de Santos.

Palavras-chave: deconvolução de Euler, dados aeromagnetométricos de alta resolução, Alto de Cabo Frio, Bacia de Santos, magmatismo alcalino.

\footnotetext{
${ }_{1}$ Universidade Federal Fluminense (UFF), Instituto de Geociências, Departamento de Geologia, Avenida General Milton Tavares de Souza s $/ n^{\circ}-4^{\circ}$ andar, Gragoatá, 24210-346 Niterói, RJ, Brasil. Tel.: (24) 2242-1230 - E-mail: andredemonte@uol.com.br

2Universidade Federal Fluminense (UFF), Instituto de Geociências, Departamento de Geologia, Avenida General Milton Tavares de Souza s/n $-4^{\circ}$ andar, Gragoatá, 24210-346 Niterói, RJ, Brasil. Tel.: (21) 2629-5929 - E-mail: adalberto@igeo.uff.br

3 Universidade Federal Fluminense (UFF), Instituto de Geociências, Departamento de Geologia, Avenida General Milton Tavares de Souza s $/ n^{\circ}-4^{\circ}$ andar, Gragoatá, 24210-346 Niterói, RJ, Brasil. Tel.: (21) 2629-5925 - E-mail: andre@igeo.uff.br
} 


\section{INTRODUÇÃO}

Para a realização deste estudo foi utilizado um conjunto de dados aeromagnetométricos de alta resolução, descritos adiante, compreendidos em área marinha do Estado do Rio de Janeiro, entre a região litorânea e aproximadamente a linha de charneira da Bacia de Santos (Fig. 1). No mapa regional desta figura é notável a anomalia isolada, Iocalizada na porção sul do mapa, a cerca de 20 km ao sul da Ilha Grande, referente a um corpo alcalino da área submersa já apontado por Zalán \& Oliveira (2005). A anomalia de Arraial do Cabo é observada somente na escala de detalhe.

Os retângulos observados no mapa regional (Fig. 1) correspondem a áreas em que se aplicou um adensamento do grid original, obtido nas duas áreas de ocorrência das anomalias produzidas pelos corpos alcalinos de Arraial do Cabo e da área submersa, que resultou na geração de dois novos mapas em escala de detalhe.

Um estudo comparativo entre estas duas anomalias magnéticas foi conduzido com abordagens qualitativa e quantitativa apoiadas pela associação dos métodos da deconvolução de Euler e amplitude do sinal analítico. A intenção deste estudo é mostrar a aplicabilidade do método da deconvolução de Euler, empregado de forma analítica e criteriosa (e não de forma indiscriminada), para obtenção de informação preliminar e vinculadora de outros métodos de inversão ou modelagem direta, utilizável na criação de modelos mais realistas de distribuição de magnetizações no ambiente geológico, principalmente em locais carentes de informação geológica direta.

Sobre a ocorrência de Arraial do Cabo (corpo aflorante), a análise é corroborada por um considerável acervo de informações geológicas (Ferrari, 2001), ao passo que a segunda é sucintamente referida na bibliografia (Zalán \& Oliveira 2005), sendo revelada somente pelos dados magnéticos.

\section{GEOLOGIA}

No contexto regional, a área se caracteriza pelos terrenos justapostos da Faixa Ribeira, de idade brasiliana, com orientação essencialmente subparalela à linha de costa. Estes terrenos foram reativados no Cretáceo Inferior, com a implantação das bacias marginais de Campos e Santos. No Cretáceo Superior, a área foi afetada por intenso magmatismo alcalino, que se estendeu até 0 Eoceno. Esse magmatismo foi acompanhado, parcialmente pela implantação do Rift Continental do Sudeste do Brasil - RCSB (Almeida, 1976; Riccomini, 1989). 0 RCSB foi revisitado por Zalán \& Oliveira (2005), que 0 denominaram Sistema de Riftes Cenozóicos do Sudeste Brasileiro, ampliando sua área de ocorrência para a porção submersa baseando-se em dados geofísicos, estendendo-0 até a charneira cretácea das bacias de Santos e Campos. Associados a este sistema de riftes, ocorrem dezenas de stocks alcalinos cujas idades neocretáceas/paleogênicas servem de balizadoras para as atividades tectônicas destes blocos altos e rebaixados. Pela análise de dados aeromagnetométricos, Zalán \& Oliveira (2005) inferem um enxame de rochas alcalinas que ocorre na área submersa rasa (plataforma). Duas destas ocorrências bordejam a linha de charneira da Bacia de Santos (Fig. 2), sendo que aquela situada mais ao norte corresponde à anomalia em foco neste trabalho.

A conhecida intrusão alcalina que ocorre nas proximidades de Arraial do Cabo (RJ), (Ferrari, 2001), ao longo do Arco de Cabo Frio, aparece como um pequeno ponto no mapa regional da Figura 2. Sua principal área de ocorrência, com pouco mais de $6 \mathrm{~km}^{2}$, está confinada à Ilha de Cabo Frio. Subordinadamente, ocorre também, em uma pequena faixa junto às Prainhas no Pontal do Atalaia e sob a forma de um pequeno plug isolado no Morro do Forno (Ferrari, 2001).

0 corpo principal, que aflora na Ilha de Cabo Frio, é alongado na direção NE-SW por cerca de 4 km e possui uma largura média de aproximadamente 2 km (Fig. 3). Segundo Ferrari (2001), este corpo é totalmente constituído por rochas alcalinas félsicas, insaturadas (nefelina sienito) a supersaturadas (quartzo sienito). Os diques associados à suíte alcalina, predominantemente félsicos, traquíticos e fonolíticos, incluem tipos menos evoluídos, como lamprófiros e basalto alcalino (Ferrari, 2001). São descritas neste contexto ocorrências de brechas com características intrusivas nas regiões de contato do corpo principal com as encaixantes regionais ou nos contatos entre diferentes litologias da suíte alcalina. Estas brechas, que englobam xenólitos mais antigos de traquito, nefelina sienito e outras rochas associadas, sugerem a recorrência de pulsos magmáticos durante a formação da suíte. As rochas encaixantes do embasamento são constituídas predominantemente por granitóides e migmatitos, com característicos metabasitos, apresentando foliação bem marcada de direção predominantemente NE-SW (Valença, 1975). Datações K-Ar em biotita forneceram idades entre 53 e $54 \mathrm{Ma}$ (Sonoki \& Garda, 1988; Thompson et al., 1998) e uma datação Ar-Ar em fonolito (rocha total) realizada por Ferrari (2001) apontou uma idade de $52.1 \pm 0.8 \mathrm{Ma}$.

\section{DADOS AEROMAGNETOMÉTRICOS}

0 aerolevantamento que gerou os dados magnetométricos utilizados neste trabalho, realizado em 2003 pela Fugro-Lasa, foi 


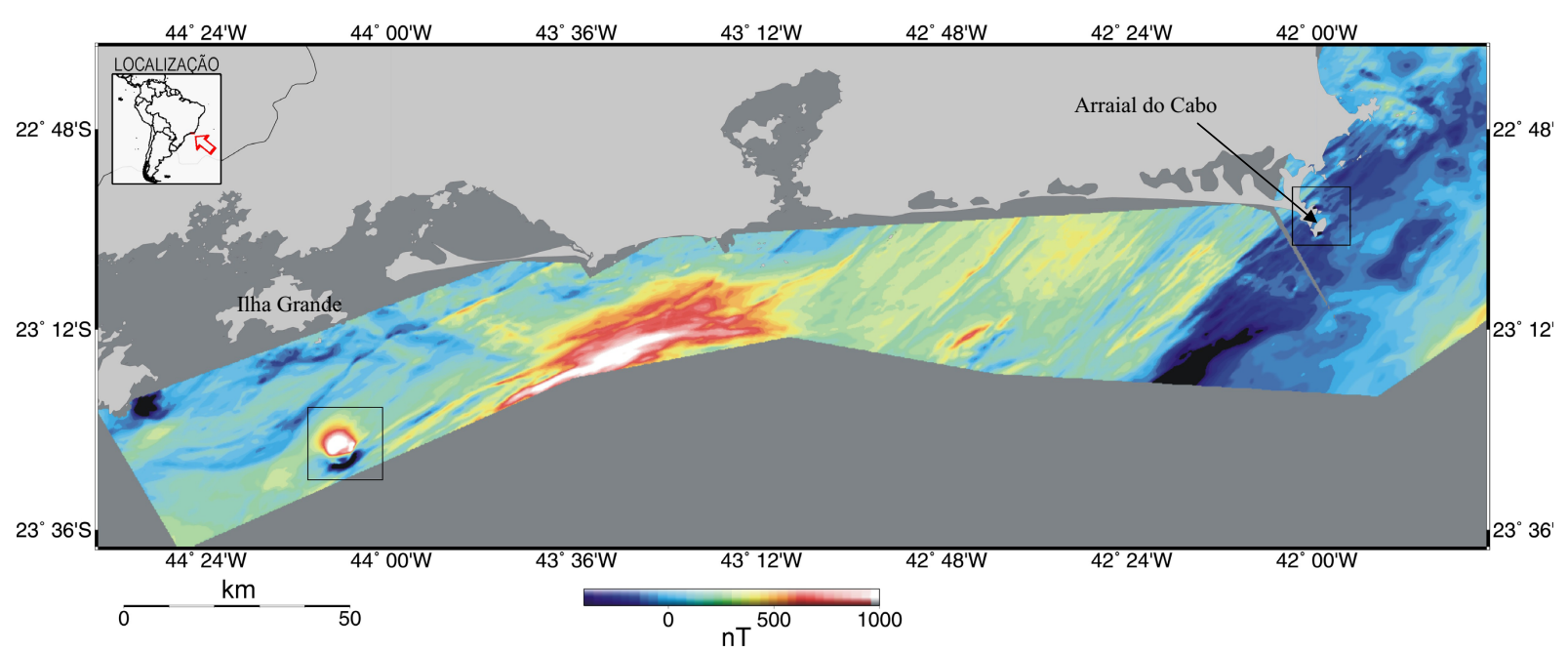

Figura 1 - Mapa do campo magnético total anômalo. Observar a notável anomalia localizada no SW da área, a SE da Illha Grande. Os retângulos a NE e SW assinalam, respectivamente, as áreas de Arraial do Cabo e submersa que foram detalhadas para aplicação da metodologia aqui proposta.

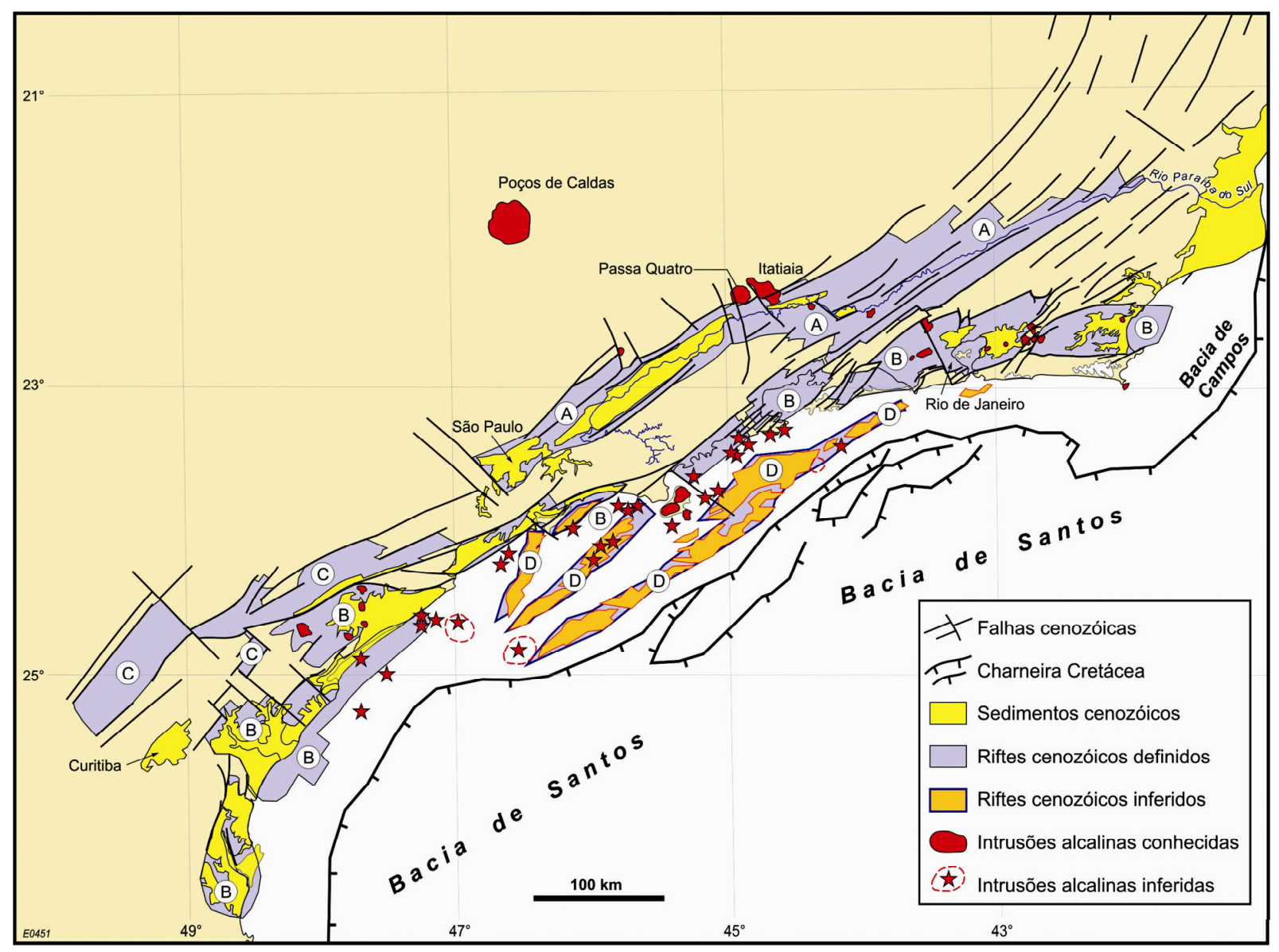

Figura 2 - Mapa regional onde se observam as diversas ocorrências de intrusões alcalinas conhecidas e inferidas através dos dados aeromagnéticos que ocorrem nas regiões proximais das Bacias de Santos e Campos (figura extraída de Zalán \& Oliveira, 2005). 
conduzido a uma altura média de vôo de 150 metros, com espaçamento de 500 metros entre linhas de produção e 2000 metros entre linhas de controle. A direção das linhas de produção foi N $30^{\circ} \mathrm{W}$.

Este conjunto de dados foi processado e disposto em maIha regular com espaçamento de 250 metros, utilizando-se um algoritmo de mínima curvatura para interpolação dos valores numéricos. A partir deste grid de dados, um mapa do campo magnético total anômalo foi gerado originalmente na escala de 1:400.000. A Figura 1 apresenta este mapa reduzido.

Um adensamento do grid original foi obtido nas duas áreas de ocorrência das anomalias produzidas pelos corpos alcalinos de Arraial do Cabo e da área submersa (Fig. 1), gerando dois novos mapas em escala de detalhe. Este adensamento foi produzido com o mesmo algoritmo de mínima curvatura referido acima, resultando em uma nova malha regular com espaçamento de 100 metros. Dada a alta qualidade dos dados e a grande precisão de seu posicionamento, acreditam os autores que a geração de artificialidades (ruído) neste processo de adensamento é irrelevante.

\section{INTERPRETAÇÃO QUALITATIVA DAS ANOMALIAS MAGNÉTICAS}

As intrusivas alcalinas de Arraial do Cabo apresentam notável resposta magnética, ostentando anomalias isoladas de grande amplitude. De forma correlata, as diversas anomalias, com características semelhantes, que ocorrem na área submersa são associadas a ocorrências não conhecidas destas rochas (Fig. 2).

A Figura 3B mostra o mapa do campo total correspondente à área de Arraial do Cabo. Sobre a província alcalina, é evidente a presença de uma região magneticamente anômala encaixada de forma discordante em um contexto regional caracterizado por marcantes alinhamentos magnéticos de direção NE-SW.

Na porção NE da Ilha de Cabo Frio se observa um alto magnético, que se estende além dos limites da mesma, limitado ao sul por uma intensa anomalia com acentuada componente negativa, com uma amplitude absoluta máxima superior a $800 \mathrm{nT}$. Ao norte do corpo principal ocorrem mais duas anomalias expressivas. A primeira, de menor amplitude, cerca de $280 \mathrm{nT}$, se encontra sobre as Prainhas e a outra, que se encontra sobre o Morro do Forno, ostenta uma grande amplitude, em torno de 700 nT.

A anomalia da área submersa, que constitui o segundo alvo deste trabalho, é observada nos mapas da Figura 4. Esta formidável anomalia circular, com uma amplitude máxima absoluta em torno de $2500 \mathrm{nT}$, é três vezes mais intensa do que a anomalia da Ilha de Cabo Frio, sendo constituída por um dipolo normal esperado para a polarização do campo atual no hemisfério sul magnético. Sobre seus flancos se sobrepõem anomalias de menor comprimento de onda relacionadas às fontes mais rasas. Estas fontes se sobressaem no mapa de amplitude do sinal analítico que será discutido mais adiante.

De forma similar à região de Arraial do Cabo, este conjunto de anomalias se posiciona discordantemente em relação às encaixantes regionais magnetizadas, que se alinham fortemente na direção NE-SW. Estes alinhamentos podem resultar das magnetizações associadas aos sistemas de falhas e fraturas que se desenvolveram na charneira cretácea da Bacia de Santos, sendo de origem diferente daqueles observados na região de Arraial do Cabo, que, apesar de se orientarem na mesma direção, se originam das estruturas pré-cambrianas da extensão submersa do Terreno Cabo Frio como definido por Heilbron et al. (2000) na área emersa.

\section{ANÁLISE QUANTITATIVA}

\section{Amplitude do sinal analítico}

$\mathrm{Na}$ análise 3D a função amplitude do sinal analítico simples, ou seu valor absoluto, definida num ponto de coordenadas $(x, y)$ pode ser deduzida a partir dos três gradientes ortogonais do campo magnético total da seguinte forma (Roest et al., 1992):

$$
|A(x, y)|=\left[\left(\frac{\partial T}{\partial x}\right)^{2}+\left(\frac{\partial T}{\partial y}\right)^{2}+\left(\frac{\partial T}{\partial z}\right)^{2}\right]^{1 / 2}
$$

onde $|A(x, y)|$ é a amplitude do sinal analítico e $T$ o campo magnético total observado no ponto $(x, y)$.

A amplitude do sinal analítico corresponde a uma função simétrica em forma de sino com seu máximo coincidindo, teoricamente, com o contato do corpo magnético ou com o seu centro quando se trata de um corpo estreito como um pequeno dique ou stock. Utilizando um algoritmo baseado na Equação 1 (Geosoft Inc.), foram gerados dois mapas (Figs. 3A e 4A) sobre as anomalias estudadas. Sua descrição se encontra mais adiante no item Interpretação dos resultados.

A Equação 1 reescrita para duas dimensões, eliminando 0 termo $(\partial T / \partial y)^{2}$, foi utilizada em rotina computacional para análise em perfil (Geosoft Inc.) para apoiar a metodologia descrita no item a seguir. 
A

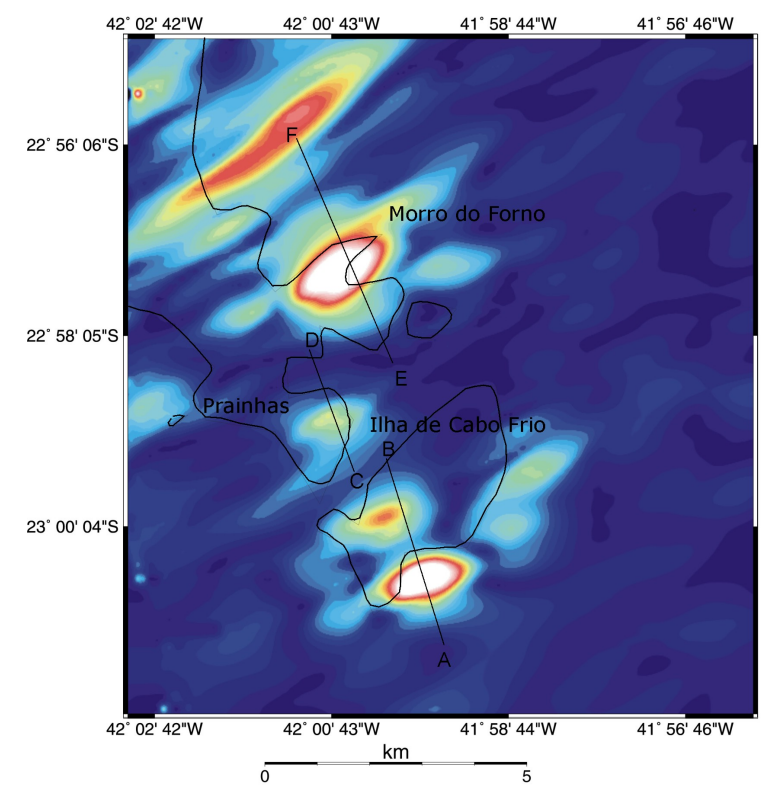

B

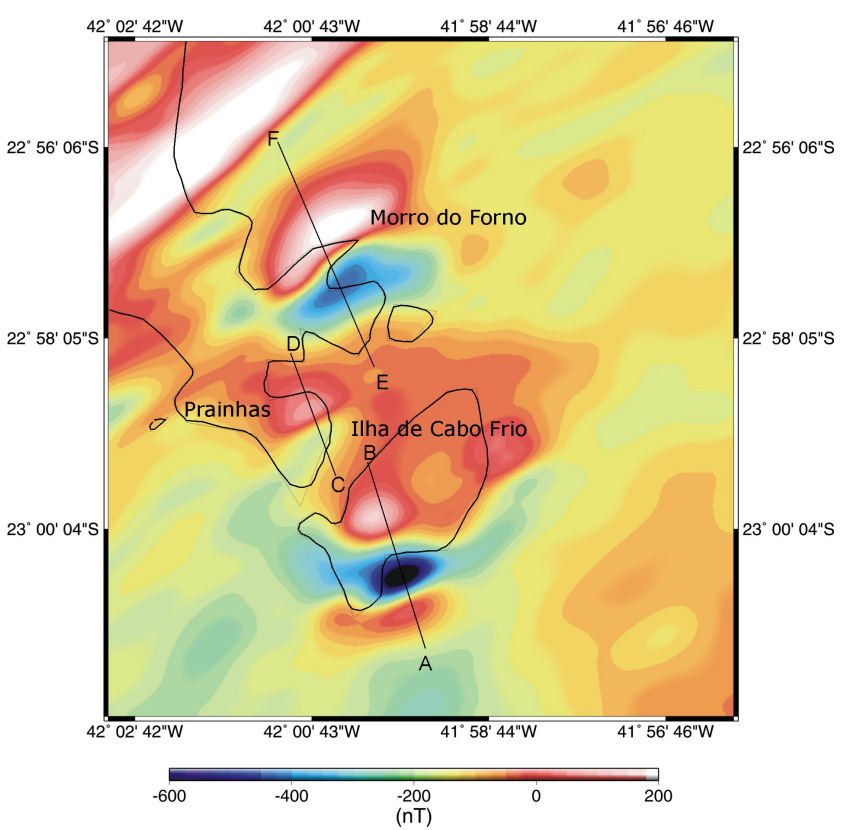

Figura 3 - Anomalia magnética da província alcalina de Arraial do Cabo. A - mapa de amplitude do sinal analítico onde os altos valores estão representados em vermelho e branco e os tons azuis representam os baixos. B - mapa de intensidade do campo total. Os perfis AB, CD e EF foram extraídos para estudo.

A

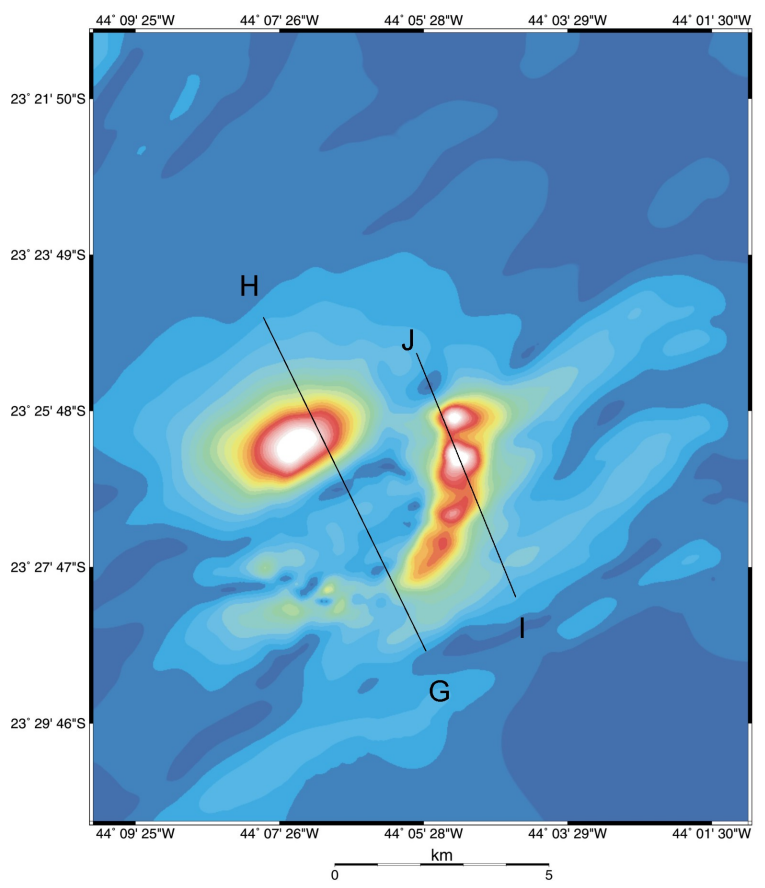

B

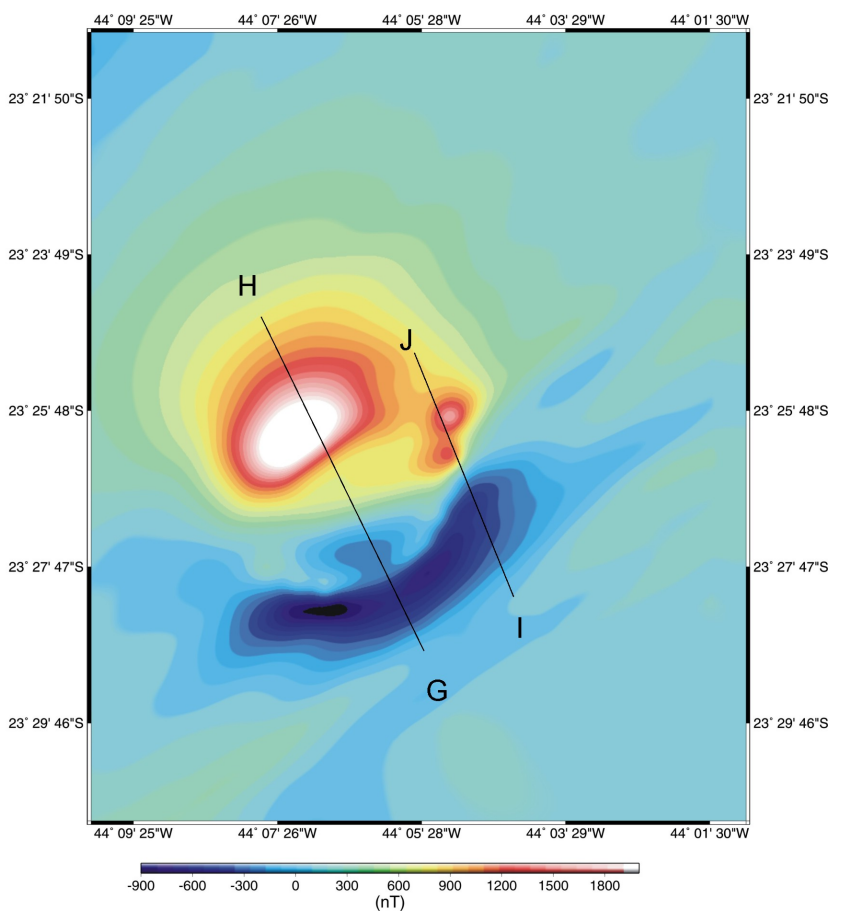

Figura 4 - Anomalia magnética da área submersa. A - mapa de amplitude do sinal analítico onde os altos valores estão representados em vermelho e branco e os tons azuis representam os baixos. B - mapa de intensidade do campo total. Os perfis GH e IJ foram extraídos para estudo. 


\section{Deconvolução de Euler}

Utilizando 0 método conhecido como deconvolução de Euler desenvolvido por Thompson (1982), os autores deste trabalho propõem a associação de três metodologias ou três critérios distintos para estimativa dos parâmetros posição, profundidade e índice estrutural das fontes magnéticas, ou seja:

- o método clássico baseado no índice estrutural tentativo que fornece o cluster mais denso de soluções, isto é, de mínimo desvio padrão (Thompson, 1982); e

- 0 índice estrutural tentativo que produz a estimativa do nível de base da anomalia magnética que apresente 0 mínimo coeficiente de correlação cruzada entre ele próprio e a anomalia (Barbosa et al., 1999).

Adicionalmente, propõem os autores o emprego da função amplitude do sinal analítico com vistas a posicionar a janela de dados no ponto de máximo gradiente da anomalia como será descrito no item a seguir.

\section{Embasamento teórico}

Thompson (1982) procurou representar as anomalias de campos potenciais através de fontes pontuais equivalentes, demonstrando que a equação de homogeneidade de Euler pode ser reescrita para fontes infinitamente longas na direção $y$ da seguinte forma:

$$
\begin{gathered}
\frac{(x-x 0) \partial \Delta T(x, z)}{\partial x}+\frac{(z-z 0) \partial \Delta T(x, z)}{\partial z} \\
=-\eta \Delta T(x, z)
\end{gathered}
$$

onde $\Delta T(x, z)$ é a anomalia do campo magnético total produzida por uma fonte nas coordenadas $(x 0, z 0)$ e $\eta$, que é conhecido como o índice estrutural, está relacionado com a natureza da fonte. Os valores de $\eta$ se relacionam com a forma da fonte de acordo com a Tabela 1.

Tabela 1 - Relação do índice estrutural com a geometria da fonte, segundo Thompson (1982).

\begin{tabular}{|l|c|}
\hline \multicolumn{1}{|c|}{ Fonte } & Índice Estrutural $-\eta$ \\
\hline Esfera & 3 \\
\hline Cilindro horizontal ou vertical & 2 \\
\hline Dique ou soleira & 1 \\
\hline Contato & 0 \\
\hline
\end{tabular}

Considerando a anomalia observada $h$, correspondente à anomalia $\Delta T$ adicionada a uma constante $b$ (nível de base), medida em um plano constante $z=0$, temos:

$$
h(x, z)=\Delta T(x, z)+b
$$

Combinando as Equações 2 e 3, obtemos:

$$
\frac{x 0 \partial h}{\partial x}+\frac{z 0 \partial h}{\partial z}+\eta b=\frac{x \partial h}{\partial x}+\eta h
$$

As soluções estimadas para os parâmetros $x 0, z 0 \mathrm{e} b$ da Equação 4 são obtidas no sentido dos mínimos quadrados por um estimador padrão que opera em cada uma das diversas janelas de dados, deslocadas através da anomalia, gerando clusters de soluções. 0 valor de $\eta$ é obtido por uma série de tentativas conforme os critérios descritos a seguir.

Barbosa et al. (1999) fazem uma análise teórica da Equação 4 e fornecem uma detalhada explanação sobre 0 comportamento dos parâmetros estimados $x 0, z 0 \mathrm{e} b$ e sua relação com 0 índice estrutural $\eta$. Sugerem, também, que a metodologia proposta inicialmente por Thompson (1982), onde diversos índices estruturais são testados com o intuito de tomar aquele que forneça o conjunto das soluções menos dispersas (isto é, aquele conjunto que forma o cluster mais denso), pode na prática apresentar incertezas devido ao ruído contido nos dados.

Foram utilizados no estudo aqui proposto não só o critério tradicional de Thompson (1982), como também o método de Barbosa et al. (1999) que lança mão de um coeficiente de correlação cruzada entre os valores da anomalia observados no centro de cada janela, e os valores do nível de base $b$ estimados para cada janela. 0 índice estrutural tentativo que fornecer o mínimo coeficiente de correlação cruzada corresponde à melhor solução.

Visando reduzir as incertezas, neste trabalho são comparados sistematicamente os dois conjuntos de soluções obtidos por estes dois distintos critérios.

Sendo o método do mínimo coeficiente de correlação cruzada eficiente quando a anomalia provém de fonte única, isto é, se relaciona a um único índice estrutural, procurou-se aqui interpretar cada anomalia de forma isolada. Uma vez que as soluções confiáveis estão associadas às janelas confinadas ao intervalo dos maiores gradientes da anomalia, região de pico da anomalia (Barbosa et al., 1999 e Silva et al., 2001), adotou-se como critério uma janela (denominada pelos autores deste trabalho de janela pivô) centralizada no máximo valor da amplitude do sinal analítico (função sino, como exposta anteriormente) calculado para anomalia em estudo. A janela pivô é deslocada a partir do ponto de máximo gradiente da anomalia em ambos os sentidos, ao longo de seu meio comprimento, visando a obtenção de clusters de soluções para posterior análise.

0 estudo teórico de Barbosa et al. (1999) demonstrou também a existência de uma relação linear entre o parâmetro $z 0$ (profundidade da fonte) e 0 índice estrutural $\eta$, 0 que torna a estimativa 
de profundidade sensivelmente dependente da escolha correta do índice estrutural. Por outro lado, $x 0$ (posição horizontal da fonte) é um parâmetro que depende somente dos gradientes, o que implica que as estimativas de $x 0$ no intervalo onde a anomalia é definida (isto é, no intervalo dos maiores gradientes) são válidas para qualquer escolha de $\eta$. Por conseguinte, 0 simples posicionamento da janela pivô fornece soluções confiáveis para $x 0$, ficando a análise focada na dispersão de $z 0$ em função da variação sistemática de $\eta$.

Baseando-se nestas propriedades, uma rotina computacional foi concebida pelos autores deste estudo, de forma a rastrear automaticamente os valores tentativos de $\eta$, até encontrar aqueles que satisfaçam as imposições dos critérios acima.

Adicionalmente, uma rotina foi implementada para a estimativa direta dos parâmetros visando compará-los às soluções obtidas pelos dois critérios anteriores. Neste caso, o índice estrutural $\eta$ não é introduzido como informação a priori através de tentativas sucessivas, mas é estimado como mais um dos parâmetros do problema inverso.

\section{Resultados obtidos}

A Figura 5 mostra saídas gráficas da rotina computacional, criada pelos autores, para a realização deste estudo, onde os c/usters de cruzes em azul correspondem às soluções de $x 0 \mathrm{e}$ $z 0$, obtidas pelo critério tradicional de Thompson (1982), e, em vermelho, as obtidas pelo método do mínimo coeficiente de correlação cruzada. As cruzes verdes correspondem às estimativas onde 0 índice estrutural é estimado como mais um parâmetro do problema inverso. Esta forma direta para estimativa dos parâmetros foi introduzida com o intuito de demonstrar 0 efeito indesejável causado pela dependência entre $z 0$ e $\eta$, demonstrando através de sua excessiva dispersão que o problema inverso, neste caso, carece de unicidade. Sendo assim, são consideradas para interpretação somente as soluções dos dois primeiros critérios, onde a condição de unicidade do problema inverso é garantida através da introdução do índice estrutural como informação a priori (Barbosa et al., 1999 e Silva et al., 2001).

Comparando os resultados obtidos para as cinco anomalias representadas na Figura 5, pode-se observar uma maior concordância entre os índices estruturais obtidos pelos dois primeiros critérios (representados em vermelho e azul), ao passo que os valores obtidos pela estimativa direta (representado em verde) diferem sistematicamente. A maior diferença encontrada $(0,6)$ entre os índices estruturais avaliados pelos dois primeiros critérios se verifica no perfil $A B$ da llha de Cabo Frio, no qual os clus- ters azul e vermelho de soluções tendem a se separar. Em tese, a estimativa do índice estrutural em ambientes geológicos complexos com múltiplas fontes interferentes permanece um problema sem solução para o método tradicional da deconvolução de Euler (Barbosa \& Silva, 2005). É possível que a discordância entre as estimativas dos índices estruturais pelos dois distintos critérios indique a presença de fontes interferentes próximas ou intenso ruído geológico.

0 modelo 2D da Figura 6, obtido através do método de Talwani (1965), mostra a anomalia teórica produzida por duas fontes magnéticas próximas cujo efeito somado produz uma anomalia similar à observada no perfil $\mathrm{AB}$ da Ilha de Cabo Frio (parte superior da figura). Este modelo está de acordo com o que é sugerido pelos resultados da deconvolução de Euler para o perfil AB (Fig. 5), ou seja, corpos de seção aproximadamente circular (2,3 obtido pelo critério azul que está próximo de 2 na Tabela 1). Não está indicado neste modelo o valor da magnetização dos corpos, uma vez que a deconvolução de Euler é insensível ao mesmo. É importante notar, entretanto, que a direção de magnetização do corpo mais raso no modelo é oposta (reversa) à direção do corpo mais profundo que é paralela ao campo atual.

0 perfil GH (Fig. 5), sobre a anomalia da área submersa, também pode ser explicado por um corpo de seção aproximadamente circular como sugerem os índices estruturais obtidos (2 e 1,9). 0 modelo da Figura 7, obtido pelo método de Talwani (1965), mostra um único corpo de seção aproximadamente circular, que poderia explicar a anomalia observada (parte superior da figura). A ondulação de menor comprimento de onda, superimposta ao flanco sul da anomalia, pode ser explicada pela presença de uma fonte mais rasa, de menores dimensões, como indica 0 modelo. Similarmente ao modelo da Figura 6, este foi concebido para uma magnetização não paralela ao campo atual.

A Figura 8 mostra a similaridade entre as anomalias observadas nos perfis CD e EF da província alcalina de Arraial do Cabo e perfil IJ da área submersa e a anomalia teórica calculada para um dique $(\eta=1)$. Pode-se notar que a anomalia produzida por um dique pode explicar os dados, como é sugerido pelos índices estruturais, próximos de 1, estimados pela deconvolução de Euler (Fig. 5) para os respectivos perfis.

\section{INTERPRETAÇÃO}

Os mapas da amplitude do sinal analítico mostrados nas Figuras 3 e 4 revelam que as anomalias do campo total observadas nos dois alvos resultam do efeito somado de mais de um corpo magnético. A anomalia de Arraial do Cabo é composta por quatro corpos 

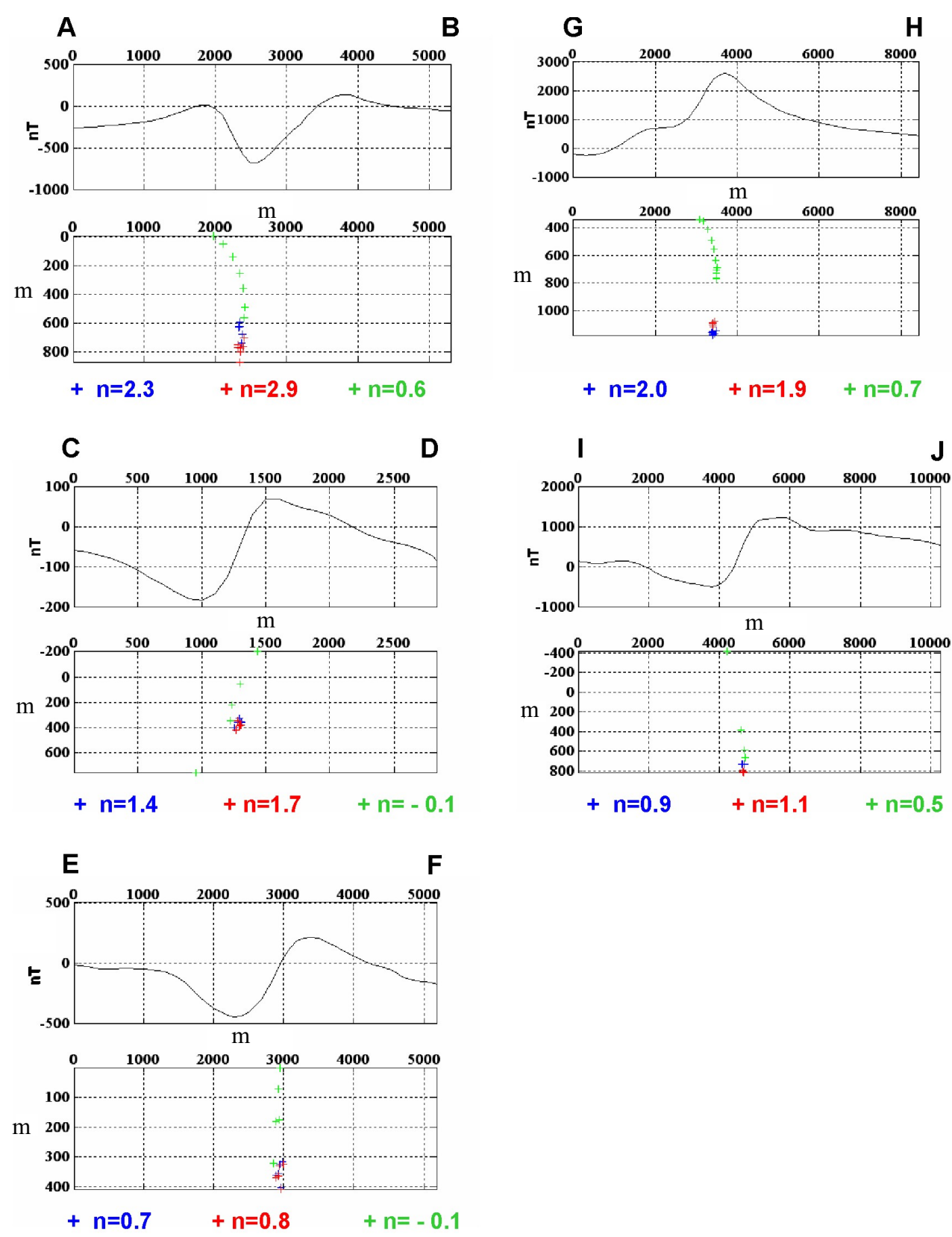

Figura 5 - Resultados obtidos pela deconvolução de Euler para os perfis AB, CD e EF de Arraial do Cabo e GH e IJ da área submersa. Abaixo de cada perfil estão os valores dos índices estruturais $\eta$ obtidos pelos três critérios descritos no texto. As cotas 0 representam o nível de vôo (150 m acima do nível do mar) e os valores negativos correspondem a soluções inconsistentes obtidas pelo terceiro critério (índice estrutural estimado como parâmetro do problema inverso) demonstrando a extrema dispersão destas soluções.

dispostos em um alinhamento NS ligeiramente curvo, que correspondem ao Morro do Forno, Prainhas e aos dois corpos a SW da Ilha de Cabo Frio que, por estarem muito próximos, formam uma única anomalia composta. 0 maciço que dá origem a esta ilha, formado predominantemente pelo nefelina e quartzo sienito aflorantes, excetuando a sua borda SW que dá origem à anomalia do perfil $A B$, apresenta uma comparativamente fraca resposta magnética.

A anomalia da área submersa é formada por um corpo principal isolado (perfil GH) e por um alinhamento NS, a leste, formado 

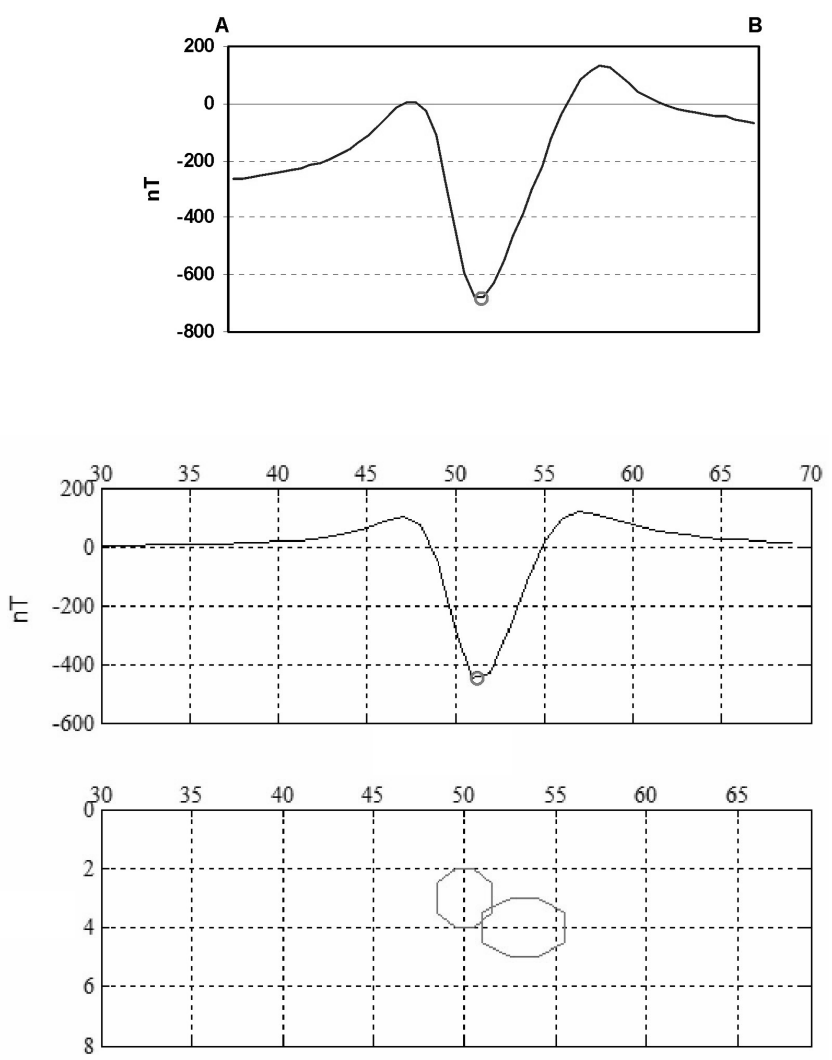

Figura 6 - Perfil AB da Ilha de Cabo Frio (topo) e anomalia teórica obtida para dois corpos de seção elíptica ou grosseiramente circular. 0 corpo mais raso apresenta magnetização reversa em relação ao campo atual cuja inclinação é igual a $36^{\circ} \mathrm{S}$. Os círculos alinham os mínimos das anomalias. Como foi assumido um caráter semiquantitativo para este modelo, não foram considerados ajustes de escala horizontal e intensidades entre a anomalia observada e teórica, ficando a análise reduzida à simples comparação da forma, distribuição dos máximos e mínimos, e os slopes entre as mesmas.

por um provável dique anelar como sugere a interpretação para 0 perfil IJ. Estes corpos apresentam comportamento similar ao alvo de Arraial do Cabo, ou seja, um corpo principal ou dois (perfil AB em Arraial) de seção aproximadamente circular como sugerem os índices estruturais próximos de 2 (cilindro) que geram as anomalias mais intensas e são circundados por corpos menores sob a forma de diques.

Tais corpos magnetizados são interpretados como intrusões constituídas por rochas de alto conteúdo de minerais ferrimagnéticos, provavelmente associadas a termos básicos (menos evoluídos) que contrastam com as demais litologias da suíte alcalina. Estas intrusões, sob a forma de plugs, ou stocks, seriam, portanto, associadas aos dutos magmáticos (pipes verticais) que deram origem ao complexo alcalino diferenciado, ou a possantes diques anelares adjacentes aos dutos principais.

$A$ análise quantitativa sugere que as anomalias dos perfis $A B$ da Ilha de Cabo Frio e GH da área submersa podem ser interpretadas como corpos magnetizados de seção aproximadamente circular (cilindro) que seriam associados aos dutos magmáticos principais. Os dois corpos da Ilha de Cabo Frio resultaram de diferentes pulsos magmáticos como sugere a hipótese interpretativa representada no modelo da Figura 6, onde 0 corpo mais raso apresenta magnetização reversa em relação ao corpo mais profundo, cuja magnetização é paralela ao campo atual.

Da mesma forma, o perfil GH da área submersa representa uma seção da anomalia provocada pelo duto magmático principal que se encontra isolado e é provavelmente mais possante do que aqueles da Ilha de Cabo Frio, uma vez que sua anomalia magnética apresenta mais do que o dobro da amplitude verificada para a anomalia da Ilha de Cabo Frio. Seu modelo (Fig. 7) também sugere a presença de magnetização não induzida pelo campo atual. Estes corpos poderiam resultar de intrusões sob a 

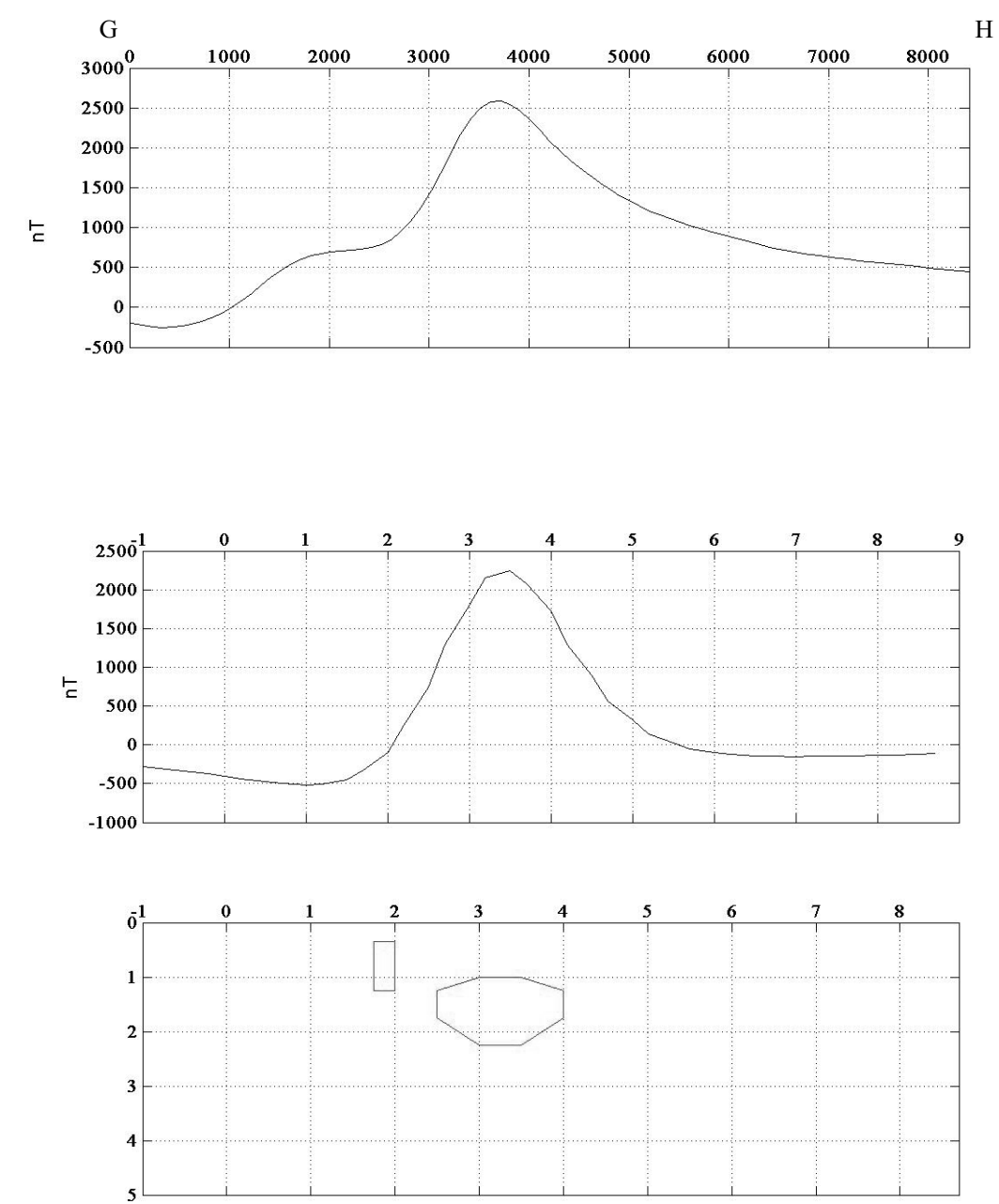

Figura 7 - Perfil GH da área submersa (topo) e anomalia teórica produzida por um corpo principal, de seção aproximadamente circular, e um corpo menor simulando a fonte que causa o ruído geológico associado à perturbação observada no flanco sul da anomalia. Este modelo foi concebido para uma magnetização não induzida pelo campo atual, isto é, magnetização total com inclinação igual a $80^{\circ} \mathrm{S}$. Como foi assumido um caráter semiquantitativo para este modelo, não foram considerados ajustes de escala horizontal e intensidades entre a anomalia observada e teórica, ficando a análise reduzida à simples comparação da forma, distribuição dos máximos e mínimos, e os slopes entre as mesmas.

forma aproximada de cúpulas ou cogumelos, como é sugerido nas Figuras 6 e 7, ou podem se encontrar simplesmente sob a forma de pipes verticais ou 'cilindros'.

Os perfis CD das Prainhas e EF do Morro do Forno parecem se associar a corpos isolados como sugere o mapa de amplitude do sinal analítico da Figura 3. No entanto, os resultados da deconvolução de Euler, principalmente para o perfil EF, apontam para diques (índice estrutural próximo de 1 na Fig. 5). A anomalia do perfil IJ da área submersa também pode ser explicada por um dique (Fig. 8). Neste caso, o mapa de amplitude do sinal analítico (Fig. 4) corrobora este resultado, mostrando a proemi- nente anomalia de um provável dique anelar situado a leste do corpo principal.

\section{CONCLUSÕES}

0 estudo comparativo realizado entre as duas anomalias revelou que existem similaridades entre as fontes interpretadas. Os mapas de amplitude do sinal analítico, em ambos os casos, revelam nitidamente a presença de corpos isolados, com expressiva magnetização, cujo efeito somado dá origem às áreas magneticamente anômalas observadas em mapa sobre a suíte alcalina de Arraial do Cabo e a área submersa respectivamente. 

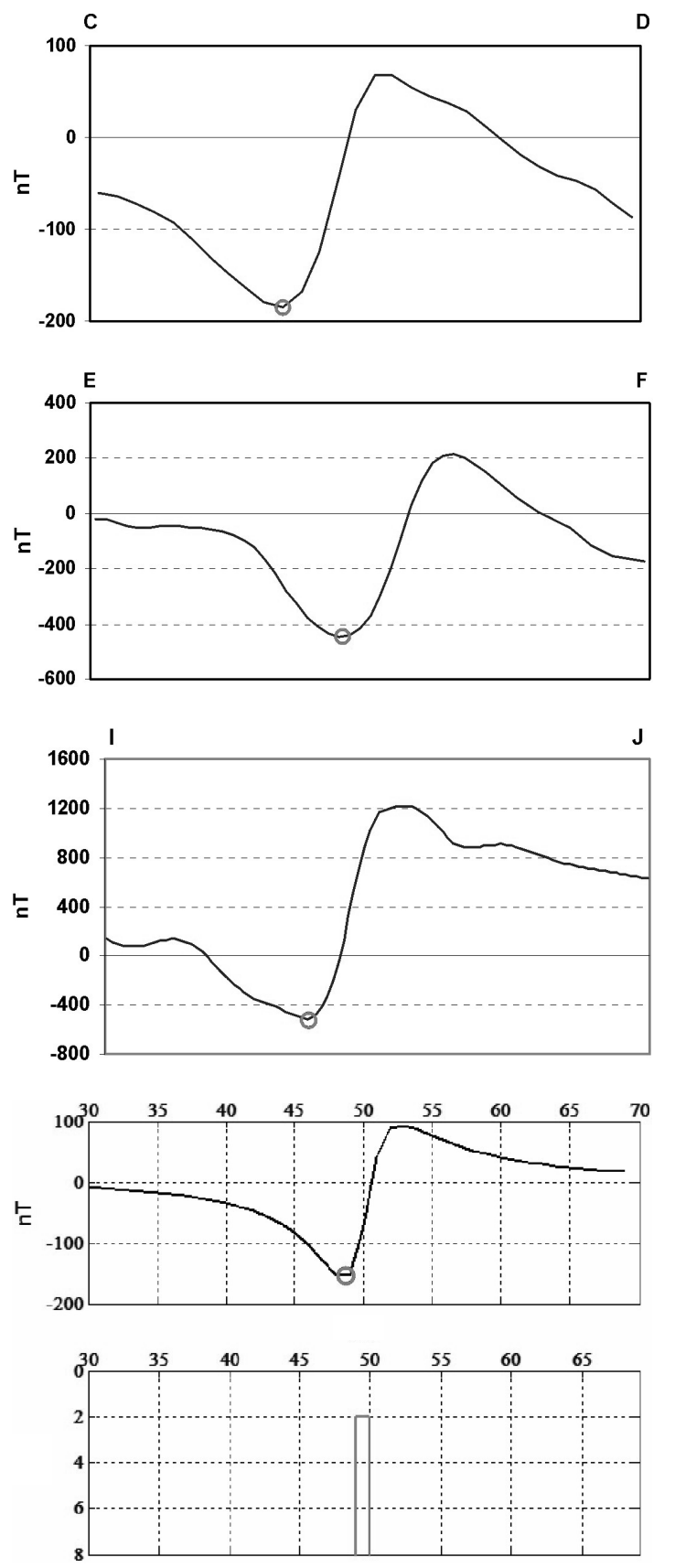

Figura 8 - Perfis CD e EF de Arraial do Cabo e IJ da área submersa e a anomalia teórica (base) calculada para um dique com a magnetização induzida pelo campo atual. Os círculos alinham os mínimos das anomalias. Como foi assumido um caráter semiquantitativo para este modelo, não foram considerados ajustes de escala horizontal e intensidades entre as anomalias observadas e teórica, ficando a análise reduzida à simples comparação da forma, distribuição dos máximos e mínimos, e os slopes entre as mesmas.

A aplicação da deconvolução de Euler, utilizando critérios para avaliação automática dos índices estruturais, produz resultados iniciais que podem orientar a construção de modelos mais realistas sobre as estruturas geológicas, mormente em ambientes carentes de informação geológica disponível como informação a priori para métodos de inversão ou modelagem.

As anomalias dos perfis $A B$ da Ilha de Cabo Frio e GH da área submersa são interpretadas como resultantes do efeito magnético dos dutos vulcânicos principais que deram origem ao complexo alcalino diferenciado. Na Ilha de Cabo Frio, estes dutos estariam adjacentes como dois corpos em forma aproximada de cúpula, cuja direção de magnetização do mais raso é reversa em relação ao campo atual, como sugere o modelo interpretativo (Fig. 6). 0 modelo do perfil GH também sugere a presença de componente de magnetização não induzida pelo campo atual.

A suíte alcalina se formou por diversos pulsos magmáticos recorrentes, como indicam as brechas mapeadas na região de Arraial do Cabo (Ferrari, 2001) . Estes pulsos, distribuídos ao longo do tempo geológico, podem conter magnetizações residuais, induzidas pelo campo reinante na época de sua cristalização. Sendo assim, o modelo da Figura 6 é geologicamente plausível, uma vez que sugere a ocorrência de um pulso, em um período de polaridade reversa do campo geomagnético. Esta é uma hipótese interpretativa relevante que merece ser verificada em futura análise comparativa, abrangendo todas as numerosas ocorrências da borda da Bacia de Santos (Fig. 2).

As anomalias dos perfis CD e EF da província alcalina de Arraial do Cabo e IJ da área submersa apresentam índices estruturais próximos a 1 , indicando que poderiam ser explicadas pelo efeito magnético de diques. Na área submersa, o perfil IJ está cortando um provável dique anelar adjacente ao corpo principal.

Os resultados da análise comparativa efetuada demonstram que 0 método de Euler é capaz de fornecer informações preliminares que orientam a construção de modelos mais completos, envolvendo distribuição de magnetizações. Entretanto, em futuros trabalhos, se faz necessária uma abordagem mais quantitativa para geração destes modelos, envolvendo não só a modelagem, como também a aplicação de métodos de inversão. Obviamente, modelos tridimensionais são mais realistas para estudar as anomalias em questão.

Acreditam os autores que um trabalho abrangente e sistemático, relacionando todas as mencionadas ocorrências de corpos alcalinos magnetizados (incluindo as exposições de geologia bem conhecida destas rochas no litoral paulista) possa contribuir para o entendimento dos processos magmáticos e tectônicos que tiveram grande influência na evolução geológica da Bacia de Santos, uma vez que tais rochas são indicadores temporais de tais processos. 0 resultado deste trabalho pode, eventualmente, fornecer alguma forma de cronologia magnética dos eventos magmáticos que afetaram esta bacia. 


\section{AGRADECIMENTOS}

Os autores agradecem à Fugro-Lasa pela cessão dos dados aeromagnéticos utilizados neste trabalho e ao PRH-ANP 11 pela concessão de bolsa de doutoramento ao primeiro autor e aos revisores anônimos que tanto contribuíram para 0 aperfeiçoamento do manuscrito.

\section{REFERÊNCIAS}

ALMEIDA FFM de. 1976. The system of continental rifts bordering the Santos Basin, Brazil. An. Acad. Bras. Ci., Rio de Janeiro, 58 (Supl.): 15-26.

BARBOSA VCF \& SILVA JBC. 2005. Deconvolução de Euler: passado, presente e futuro - um tutorial. Revista Brasileira de Geofísica, 23(3): 243-250.

BARBOSA VCF, SILVA JBC \& MEDEIROS WE. 1999. Stability analysis and improvement of structural index estimation in Euler deconvolution. Geophysics, 64: 48-60.

FERRARI AL. 2001. Evolução tectônica do Graben da Guanabara. Tese de Doutorado, IGc/USP, São Paulo. 412 p.

HEILBRON M, MOHRIAK WU, VALERIANO CM, MILANI EJ, ALMEIDA J \& TUPINAMBÁ M. 2000. From Collision to Extension: The Roots of the Southeastern Continental margin of Brazil. In: MOHRIAK W \& TALWANI M (Ed.). Atlantic Rifts and Continental Margins, AGU Geoph. Mon. 115: $1-32$.

RICCOMINI C. 1989. O Rift continental do sudeste do Brasil. Tese de
Doutorado, IGc/USP, São Paulo. 256 p.

ROEST WR, VERHOEF V \& PILKINGTON M. 1992. Magnetic interpretation using the 3-D analytic signal. Geophysics, 57: 116-125.

SILVA JBC, BARBOSA VCF \& MEDEIROS WE. 2001. Scattering, symmetry, and bias analysis of source-position estimates in Euler deconvolution and its practical implications. Geophysics, 66: 1149-1156.

SONOKI IK \& GARDA GM. 1988. Idades K-Ar de rochas alcalinas do Brasil Meridional e Paraguai Oriental: compilação e adaptação às novas constantes de decaimento. Bol. IG-USP, Série Científica, 19: 63-85.

TALWANI M. 1965. Computation with the help of a digital computer of magnetic anomalies caused by bodies of arbitrary shape. Geophysics, 30: $797-817$.

THOMPSON DT. 1982. EULDPH: A new technique for making computerassisted depth estimates from magnetic data. Geophysics, 47: 31-37.

THOMPSON RN, GIBSON SA, MITCHELL JG, DICKIN AP, LEONARDOS OH, BROD JA \& GREENWOOD JC. 1998. Migrating Cretaceous-Eocene magmatism in the Serra do Mar alkaline province, SE Brazil: Melts from the deflected Trindade Mantle Plume? J. Petrol., 39(8): 1493-1526.

VALENÇA JG. 1975. Rochas alcalinas do Estado do Rio de Janeiro correlações geológicas. Rev. Min. Met., 336: 6-11.

ZALÁN PV \& OLIVEIRA JAB. 2005. Origem e evolução estrutural do Sistema de Riftes Cenozóicos do Sudeste do Brasil. Bol. Geoc. Petrobras, Rio de Janeiro, 12: 269-300.

\section{NOTAS SOBRE OS AUTORES}

André Etienne Pacifico Peçanha Demonte Ferraz. Graduado em Geologia pela UFRRJ em 1981, realizou ao longo de 12 anos, como geofísico, dezenas de projetos de consultoria para aquisição, processamento e interpretação de dados magnetométricos, gamaespectrométricos (terrestres e aéreos), gravimétricos, EM e sísmica rasa destinados à prospecção mineral e à indústria do petróleo. Depois de 10 anos afastado da Geofísica retorna desenvolvendo tese de doutorado (pela UFF em andamento) sobre interpretação de dados aeromagnetométricos. Se concentra na pesquisa e aplicação de métodos de inversão a problemas reais de campos potenciais, envolvendo situações complexas com múltiplas fontes interferentes, magnetizações remanescentes e carência de informação a priori.

Adalberto da Silva. Graduado em Geologia pela Universidade de São Paulo em 1981. Atuou como geólogo da Petrobras em Macaé (RJ) de 1986 a 1990. Mestre em Geologia e Geofísica Marinha pela Universidade Federal do Rio de Janeiro em 1992 e Doutor em Geofísica pela Universidade de São Paulo em 1999. Em 2000, desenvolveu projeto de pós-doutorado em tratamento espectral de dados geofísicos de poço na Universidade Estadual de Campinas. Atuou como pesquisador-visitante do Programa de Recursos Humanos da ANP - PRH 11 - Departamento de Geologia/LAGEMAR/UFF de 2001 a 2005, ingressando neste departamento em 2005 como Professor Adjunto. Atua na área de Geofísica Aplicada ao Petróleo, Geologia e Geotectônica e Avaliação de Formações.

André Luiz Ferrari. Graduado em Geologia pela UFRJ, em 1978, e com Doutorado em Geologia Sedimentar pela USP, em 2001, tem atuado na análise estrutural da reativação de áreas plataformais em bordas continentais, formação de bacias rifte, tectono-sedimentação e controle tectônico do magmatismo. Desde 1984 é Professor do Departamento de Geologia da UFF. Atualmente é Diretor do Instituto de Geociências da UFF. 